\title{
Grupo Colaborativo de Resistencia Bacteriana, Chile: recomendaciones 2014 para el control de la resistencia bacteriana
}

\author{
Marcela Cifuentes, Francisco Silva, J. Miguel Arancibia, Ruth Rosales, M. Cristina Ajenjo, Gisela Riedel, \\ Rossana Camponovo y Jaime Labarca, en representación del Grupo Colaborativo de Resistencia Bacteriana*
}

\section{Grupo Colaborativo de Resistencia Bacteriana, Chile: recommendations 2014 towards the control of bacteria resistance}

Five issues were reviewed in depth at the 2014 annual meeting of Colaborative Group Against Bacterial Resistance and the antecedents and conclusions are detailed in this document. I.- News in CLSI 2014: the difficulties and implications on its implementation at the local level were reviewed and recommendations were set. II.- Criteria for determining the incidence of multi-resistant microorganism in critical care units where indicators and monitoring methodology for better quantification of microorganisms were defined. III.- Quality requirements were established to be considered by the professionals involved in the selection of antimicrobials in the hospital. IV.- Transfer policies, screening and contact precautions for the control of transmission of multiresistant bacteria. V.- Recommendations for health facilities when a carbapenemase producing enterobacteriacea is detected, in a checklist format for rapid deployment in hospitals without endemia of these agents. These are suggestions that arise from the joint work of specialists from many hospitals that do not represent consensus or recommendation, but may help to control the resistance level of each health facility in the country.

Key words: antimicrobial resistance, susceptibility testing, carbapenemasa, surveillance.

Palabras clave: resistencia antimicrobiana, pruebas de susceptibilidad, carbapenemasa, vigilancia.

\section{Introducción}

$\mathrm{E}$ 1 Grupo Colaborativo de Resistencia Bacteriana (GCR) de la Sociedad Chilena de Infectología ha realizado 10 reuniones anuales. En estos encuentros participa un número aproximado de 70 profesionales (microbiólogos, infectólogos y químico-farmacéuticos) representando a un número cercano a 40 hospitales del país, que incluye establecimientos públicos, privados e institucionales, hospitales generales, de adultos y pediátricos, de Santiago y otras Regiones, abarcando desde Antofagasta a Punta Arenas, Magallanes. Desde sus inicios, en estas reuniones se han realizado talleres de discusión de problemas relacionados a la resistencia bacteriana, que incluyen aspectos microbiológicos, clínicos, epidemiológicos y de prevención. Estos talleres se constituyen cada año por grupos de profesionales de las diversas áreas involucradas y permiten discutir temas con una mirada multidisciplinaria y desde la realidad local de los distintos hospitales.

El año 2014 fue la décima versión de la Reunión Anual de este grupo y se realizaron cinco talleres con temas de gran relevancia para los hospitales. Consideramos que las
Hospital Clínico San Borja Arriarán. Laboratorio de Microbiología (MC). Hospital Clínico Universidad de Chile. Servicio de Laboratorio Clínico y CPC-IAAS (MC, FS). Hospital San Juan de Dios. Unidad de Infectología (JMA). Hospital Barros Luco Trudeau. Unidad de Farmacia (RR). Pontificia Universidad Católica de Chile. Departamento de Enfermedades Infecciosas del Adulto, Escuela de Medicina (MCA, JL).

Hospital Clínico Guillermo Grant Benavente. Unidad de Infectología (GR). Laboratorio Integramédica (RC)

Recibido: 20 de marzo de 2015

Correspondencia a: Jaime A. Labarca Labarca jlabarca@med.puc.cl

\footnotetext{
*Grupo Colaborativo de Resistencia Bacteriana

Integrantes Taller 1: Silva Francisco, Camponovo Rossana, Álvarez María Isabel, Bello Helia, Briceño Isabel, Cruz María Carolina, Domínguez Mariana, Fernández Alejandra, García Patricia, González Gerardo, Guzmán Daniel, Illesca Vijna, Joyas Alejandro, Juliet Chrystal, Mühlhauser Margareta, Peralta Gerardo, Porte Lorena, Rioseco María Luisa, Rojas Pamela, Rocha Freddy, Sakurada Andrea.

Integrantes Taller 2: Cifuentes Marcela, Álvarez J, Blamey Rodrigo, Cona Erna, Dabanch Jeannette, Gálvez Ricardo, Legarraga Paulette, Pavez Mónica, Salgado Fabiola.

Integrantes Taller 3: Rosales Ruth, Bavestrello Luis, Benadof Dona, Acuña Mirtha, Álvarez Ana María, Barthel Elizabeth, Chaboutti Henriette, Cofre José, Coria Paulina, Domínguez Isabel, Jensen Werner, Luppi Mario, Morales Jorge, Pavez Daniela, Rojas Álvaro, Usedo Pedro, Villena Rodolfo, Aylwin Mabel.

Integrantes Taller 4: Ajenjo María Cristina, Riedel Gisela, Delama Ignacio, Diomedi Alexis, Lafourcade Mónica, Llancaqueo Álvaro, Marín Gloria, Muñoz Rodrigo, Pérez Jorge, Salvador Francisco, Stegmeier Helen.

Integrantes Taller 5: Arancibia José Miguel, Bustamante Claudia, Castelli Anna, Concha Carla, Cornejo Andrés, De la Lastra Virginia, Díaz Horacio, Galindo Carolina, Muñoz Rubén, Olivares Roberto, Tapia Jaime, Tinoco Javier.
} 
conclusiones de estos talleres pueden ser de interés general en el país y su difusión podría ayudar a los distintos establecimientos, no pretendiendo ser un consenso o tener rol de guías nacionales. Los talleres trataron temas relevantes de diagnóstico, vigilancia epidemiológica y prevención de la resistencia bacteriana. Estos fueron:

- Novedades del CLSI 2014. Recomendaciones y dificultades en la implementación de puntos de corte para bacilos gramnegativos.

- Criterios para la determinación de incidencia de microorganismos multi-resistentes en unidades críticas.

- Requisitos de calidad de los antimicrobianos. Lo que los programas de uso de antimicrobianos deben saber.

- La transmisión en el control de la resistencia, políticas de traslados, tamizaje y precauciones de contacto.

- Recomendaciones para los establecimientos de salud, frente a informe de paciente con enterobacteria productora de carbapenemasa.

\section{Taller 1}

Novedades del CLSI 2014.

Recomendaciones y dificultades

en la implementación de puntos

de corte para bacilos gramnegativos

En este taller se discutieron puntos clave en la implementación de las nuevas recomendaciones para el estudio de susceptibilidad antimicrobiana del año 2014 del documento CLSI M100-S24 ${ }^{1}$. Se consideró su aplicabilidad en los laboratorios nacionales considerando tanto aspectos técnicos como implicancias clínicas. Los puntos que se discutieron fueron cuatro y se estructuraron en el documento como "cambio", "problema" y "recomendaciones del grupo".

\section{Cefazolina y cefalotina en enterobacterias}

Cambios:

- CLSI recomienda implementar nuevos puntos de corte para cefazolina en aislados de orina en pacientes con infección del tracto urinario (ITU) no complicada. Cefazolina sería el antimicrobiano que representa a: cefalexina, loracarbef, cefdinir, cefuroxima y cefpodoxima. En caso de resultados resistente, se pueden probar cada antimicrobiano por separado ya que hay cepas resistentes a cefazolina y susceptibles a otras cefalosporinas.

- La recomendación anterior reemplazaría el uso de cefalotina como agente que representa la susceptibilidad a cefadroxilo y otras cefalosporinas orales.

\begin{tabular}{|l|c|c|c|}
\hline $\begin{array}{l}\text { Cefazolina } \\
\text { Puntos de corte }\end{array}$ & $\begin{array}{c}\text { Sensible } \\
(\boldsymbol{\mu} \mathbf{g} / \mathbf{m L})\end{array}$ & $\begin{array}{c}\text { Intermedio } \\
(\boldsymbol{\mu} \mathbf{g} / \mathbf{m L})\end{array}$ & $\begin{array}{c}\text { Resistente } \\
(\boldsymbol{\mu} \mathbf{g} / \mathbf{m L})\end{array}$ \\
\hline Orina (ITU no complicada) & $\leq 16$ & - & $\geq 16$ \\
\hline Otras muestras & $\leq 2$ & 4 & $\geq 8$ \\
\hline
\end{tabular}

\section{Problemas:}

- El nuevo punto de corte para cefazolina no incluye la predicción de actividad de cefadroxilo. No hay estudios para este antimicrobiano ya que no se utiliza en E.U.A. donde se generó la recomendación (Comunicación personal Dra. E. Palavecino, Wake Forest University Health Sciencies Medical Center, Winston-Salem, NC).

- La mayoría de los laboratorios no tienen la suficiente información clínica para definir si se trata de un aislado obtenido de un paciente con ITU no complicada (se excluyen hombres, mujeres embarazadas y postmenopáusicas, signos de ITU alta).

- De utilizar el nuevo punto de corte en todos los aislados urinarios puede producirse un informe sensible en orina (por ej. CIM $8 \mu \mathrm{g} / \mathrm{mL}$ ) y resistente en sangre; si se tratara de un caso complicado, generaría problemas clínicos ya que se estaría informado sensible a agentes orales y resistente a cefazolina (ev).

Recomendaciones y acuerdos del grupo:

- No realizar el cambio de utilizar cefazolina como agente que representa a cefalosporinas de primera generación orales.

- Mantener durante el año 2014 el uso de cefalotina con los puntos de corte vigentes actualmente, aunque se reconoce que éstos también son válidos para ITU no complicada, pero extrapolables a cefadroxilo. Además, generan un número importante de resultados intermedios.

- En casos especiales (mujeres embarazadas, ITU que se sepan complicadas, otros aislados no urinarios) se debe mantener el uso e informe de cefazolina con los puntos de corte vigentes.

\section{$\beta$ - lactamasas de espectro extendido-BLEE}

\section{Cambio:}

- No hay cambio en CLSI 2014; se mantiene que clínicamente no es necesario testear BLEE ni editar los resultados si se utilizan los puntos de corte propuestos del 2010 en adelante.

\section{Problema:}

- Se consulta entre los asistentes y todos hacen prueba de confirmación de BLEE y edición de resultados.

- En Chile, considerando la epidemiología y las medidas de contención a adoptar, es necesario mantener la detección de BLEE.

- La evidencia acumulada no es categórica en demostrar que cepas BLEE positivas pueden ser tratadas con cefalosporinas de tercera generación si los puntos de corte se encuentran dentro del rango susceptible ${ }^{2}$. 
Recomendaciones y acuerdos de este grupo:

- En consideración de la seguridad de los pacientes y el riesgo de falla terapéutica en este contexto, se acuerda mantener el estudio fenotípico confirmatorio de BLEE y, según sus resultados, editar el informe de susceptibilidad a las cefalosporinas. Similares recomendaciones han emanado de instituciones extranjeras como CIDEIM, Colombia ${ }^{3}$ e Instituto Malbrán, Argentina.

- No informar ceftazidima en cepas de enterobacterias para focalizar su uso en Pseudomonas aeruginosa (práctica clínica habitual en nuestro país).

- Estas recomendaciones deben ser revisadas anualmente considerando la epidemiología local y la nueva evidencia disponible.

\section{Susceptibilidad Dosis Dependiente (SDD) para cefepime y cambio en puntos de corte para enterobacterias}

\section{Cambio:}

- CLSI crea este año una categoría SDD sólo para cefepime de manera de indicar su utilidad en cierto rango de CIM en las cuales la dosis del antimicrobiano podría aumentarse para lograr efecto terapéutico, indicándose además que los laboratorios registren -a pie de página- la dosis sugerida para utilizar frente a este nuevo criterio.

- Además se modifican los puntos de corte para este antimicrobiano como se indica en la siguiente tabla:

\begin{tabular}{|l|c|c|c|c|}
\hline $\begin{array}{l}\text { Cefepime } \\
\text { Puntos de } \\
\text { corte }\end{array}$ & $\begin{array}{c}\text { Sensible } \\
(\mu \mathrm{g} / \mathrm{mL})\end{array}$ & $\begin{array}{c}\text { Intermedio } \\
(\mu \mathrm{g} / \mathrm{mL})\end{array}$ & $\begin{array}{c}\text { SDD } \\
(\mu \mathrm{g} / \mathrm{mL})\end{array}$ & $\begin{array}{c}\text { Resistente } \\
(\mu \mathrm{g} / \mathrm{mL})\end{array}$ \\
\hline 2013 & $\leq 8$ & 16 & - & $\geq 32$ \\
\hline 2014 & $\leq 2$ & - & $4-8$ & $\geq 16$ \\
\hline
\end{tabular}

\section{Problema:}

- No está clara la capacidad de los sistemas informáticos de laboratorio (SIL) para implementar una nueva categoría interpretativa para un único antimicrobiano.

- No existe una manera fácil y rápida de difundir el significado de SDD en los equipos clínicos ya que hasta la fecha este concepto está implícito en la categoría de susceptibilidad intermedia.

- No parece adecuado que los laboratorios pongan -a pie de página- la dosificación sugerida según este criterio ya que es una decisión clínica dependiente también de otros factores.

Recomendaciones y acuerdos de este grupo:

- En nuestro país el uso de cefepime no es masivo y en general en cepas productoras de BLEE se prefieren otras alternativas terapéuticas.
- El cambio a SDD podría tener algún uso en cepas productoras de BLEE pero dado que este resultado aún lo editamos, no tendrá repercusión en informe.

- Se recomienda implementar los nuevos puntos de corte para así detectar un bajo número de cepas resistentes que previamente podrían haber resultado intermedias por un mecanismo distinto de BLEE (cada hospital puede obtener el dato del cambio esperado en susceptibilidad si dispone de las CIM o halos en $\mathrm{mm}$ ).

- Las cepas que den resultado en SDD informarlas por ahora como intermedias. Esto también ha sido sugerido por CLSI cuando no se puede implementar el SDD.

- Los sistemas automatizados Vitek ${ }^{\circledR}$ y Phoenix ${ }^{\circledR}$ tienen rango de lectura adecuado para implementar estos nuevos puntos de corte.

Cambio de puntos de corte para carbapenémicos en Acinetobacter baumannii

Cambio:

- Se modifican los puntos de corte para carbapenémicos en A.baumannii.

\begin{tabular}{|l|c|c|c|c|}
\hline & Año & $\begin{array}{c}\text { Sensible } \\
(\boldsymbol{\mu} \mathbf{g} / \mathbf{m L})\end{array}$ & $\begin{array}{c}\text { Intermedio } \\
(\boldsymbol{\mu} \mathbf{g} / \mathbf{m L})\end{array}$ & $\begin{array}{c}\text { Resistente } \\
(\boldsymbol{\mu} \mathbf{g} / \mathbf{m L})\end{array}$ \\
\hline \multirow{2}{*}{ Imipenem } & 2013 & $\leq 4$ & 8 & $\geq 16$ \\
\cline { 2 - 5 } & 2014 & $\leq 2$ & 4 & $\geq 8$ \\
\hline \multirow{2}{*}{ Meropenem } & 2013 & $\leq 4$ & 8 & $\geq 16$ \\
\cline { 2 - 5 } & 2014 & $\leq 2$ & 4 & $\geq 8$ \\
\hline
\end{tabular}

Recomendaciones y acuerdos:

- Se recomienda implementar el cambio, tanto para métodos de dilución (CIM) como difusión (discos).

- Considerar que éste se basa en determinados esquemas de dosificación, por lo que esquemas distintos deben ser de resorte clínico, conociendo las limitaciones de los puntos de corte.

- Los sistemas automatizados tienen un rango de lectura adecuado para implementar el cambio.

\section{Taller 2}

\section{Criterios para la determinación}

de incidencia de microorganismos multi-resistentes en unidades críticas

La determinación de los porcentajes de susceptibilidad bacteriana a los antimicrobianos no siempre se correlaciona bien con la endemia hospitalaria y las infecciones asociadas. Por ejemplo, se puede tener una bacteria con $100 \%$ de resistencia a un determinado antimicrobiano (por ej., carbapenémicos) pero su frecuencia es tan baja que aparece en forma esporádica en la institución y por ende, no es un problema epidemiológico. Debido a ello, 
se hace necesario recomendar la determinación de un indicador que refleje la situación epidemiológica local, siendo recomendada la incidencia de aquellas bacterias con resistencia epidemiológicamente relevantes, especialmente en unidades de cuidados intensivos, usando un sistema estandarizado ${ }^{4,5}$.

En consecuencia, es conveniente complementar la vigilancia de resistencia bacteriana actual con una vigilancia basada en evaluar la incidencia de bacterias con resistencia específica o "densidad de incidencia", indicador que se basa en el $\mathrm{N}^{\circ}$ de casos de infección y/o colonización por 1.000 días/cama ocupada del servicio o institución analizada, en un determinado período de tiempo.

El indicador se puede obtener por dos métodos: uno de ellos es la incidencia de casos de infecciones que da cuenta del impacto clínico de la resistencia y el otro es la incidencia de bacterias resistentes (colonizaciones e infecciones) que dé cuenta de la endemia. El indicador ideal para la aplicación clínica es el que refleja la incidencia de casos verdaderos de infección por el microorganismo con la resistencia que se quiere estudiar, pero este numerador es difícil de obtener y requiere la definición estándar de cada una de las infecciones y/o un único observador clínico que evalúe la totalidad de los casos sospechosos.

En el caso de los equipos de control de infecciones y laboratorio de microbiología, la utilización de la densidad de incidencia de "ocurrencia" o "colonización/infección" es suficiente para la toma de medidas epidemiológicas. El numerador es más fácil de obtener ya que se parte del $\mathrm{n}^{\circ}$ de cultivos positivos con el microorganismo con la resistencia que se desea estudiar y, por lo tanto, más fácil de conseguir en la mayoría de los hospitales.

Para ambos indicadores el procedimiento de obtención de datos es similar:

- Periodicidad: Se recomienda emitir al menos un informe anual (1 de enero a 31 de diciembre) que permita que al establecimiento compararse con otros hospitales. Esto no excluye llevar un indicador por períodos más cortos (ej. trimestral o mensual) para que el establecimiento pueda tener un mejor control epidemiológico de sus casos.

- Dónde: Se recomiendan partir con las Unidades de Cuidados intensivos; en algunos centros se puede incluir las unidades coronarias si es que tiene una epidemiología similar a la UCI o existe una alta frecuencia de traslados entre dichas unidades. En un segundo tiempo, se pueden incluir más servicios.

- Qué vigilar: El número de ciertas infecciones causadas por determinados microorganismos. En general, para esta vigilancia se utiliza las definiciones de la vigilancia epidemiológica. En el caso que no se puede obtener información depurada de cuáles son los casos de infección, se puede construir el indicador considerando el primer cultivo positivo del agente a vigilar.

\section{Qué microorganismos vigilar:}

En ambos casos se sugiere considerar: Staphylococcus aureus resistente a meticilina (SARM), Enterococcus resistente a vancomicina (ERV), Klebsiella pneumoniae resistente a carbapenémicos, Acinetobacter baumannii resistente a carbapenémicos y $P$. aeruginosa resistente a carbapenémicos. Infecciones por otros microorganismos quedarían a discreción de cada establecimiento.

\section{Cómo obtener la información:}

- Incidencia de casos (infección/colonización) por microorganismos resistentes.

- Se sugiere que el laboratorio de microbiología confeccione un listado a partir de sus bases de datos con los cultivos positivos de cada mes que cumplen con el requisito de inclusión (ver "qué"). Una forma fácil de realizar el listado es exportando la base de datos a Whonet $^{7-8}$, y utilizar la sección de análisis de datos del mismo programa.

- En la unidad de estadísticas conseguir el $\mathrm{N}^{\circ}$ de días camas ocupadas de la unidad analizada.

- El indicador se calcula:

$N^{\circ}$ de pacientes con microorganismo resistente en período de tiempo (ej. año 2014) x 1.000

Total de días cama ocupada en UPC en mismo período de tiempo

- Una vez calculado el indicador el laboratorio centralizará el envío de la información al GCR, en caso de ser un participante activo.

- Incidencia de infecciones por microorganismos resistentes.

- Para construir el indicador de incidencia de infecciones, el listado de cultivos del punto anterior debe ser analizado por el equipo clínico (infectólogo e intensivista) para definir qué pacientes han hecho una infección a partir de ese cultivo positivo y atribuirlo o descartarlo. Lo anterior incluye muchas otras infecciones que no están consideradas por el Sistema de Vigilancia Epidemiológica de las IAAS del Ministerio de Salud.

- En la unidad de estadísticas conseguir el $\mathrm{N}^{\circ}$ de días cama ocupadas de la unidad analizada.

- Con el listado de las verdaderas infecciones causadas por el microorganismo en estudio se debe construir el indicador:

\section{$\mathrm{N}^{\circ}$ de infecciones causadas por microorganismo en período de tiempo (ej. año 2014) x 1.000}

Total de días cama ocupada en UPC en mismo período de tiempo 
- El equipo clínico debería enviar un numerador depurado (sólo pacientes con infecciones clínicas, descartando las colonizaciones) al laboratorio de microbiología para centralizar la información.

- Una vez calculado el indicador, centralizará el envío de la información al GCR, en caso de ser un participante activo.

\section{Consideraciones:}

- Incluir sólo los cultivos tomados con intención de diagnóstico y excluir aquellos derivados de programas de vigilancia (ej. Vigilancia mensual y/o de traslados de ERV).

- Se considerará como microorganismo R a carbapenémicos a cualquiera que sea resistente a uno o más carbapenémicos. No es necesario que sea $\mathrm{R}$ a todos a la vez.

- Cada establecimiento debe definir si el indicador lo muestra en forma trimestral, semestral o anual; sin embargo, para no acumular revisión de pacientes, se sugiere que el listado el laboratorio lo emita mensualmente de modo de tener que revisar pocos cultivos/ pacientes/infecciones cada vez.

- Las funciones de los diferentes roles son una sugerencia del grupo en base a su experiencia. Cada establecimiento debe definir su flujo de datos de modo de optimizar el tiempo que utilizarán, como también asegurar la generación de resultados confiables.

- Se sugiere el uso de una base de datos única en formato Excel o Access y emitir el informe en PDF.

\section{Taller 3}

Requisitos de calidad de los antimicrobianos. Lo que los programas de uso de antimicrobianos debiesen solicitar

El objetivo de este taller fue identificar y unificar los criterios de calidad que deberían ser considerados para la adquisición de antimicrobianos y los reglamentos que enmarcan este proceso. Se revisan los estudios solicitados por el Instituto de Salud Pública (ISP) al momento del registro de un determinado producto farmacéutico.

\section{Antecedentes legales}

En el Decreto Supremo 3/20109: Aprueba el Reglamento del Sistema Nacional de Control de los Productos Farmacéuticos de uso Humano, "D. Requisitos de Seguridad y Eficacia”, se destacan los siguientes puntos.

\subsection{Artículo $36^{\circ}$, de seguridad y eficacia}

Toda solicitud de registro sanitario deberá incluir antecedentes de seguridad y eficacia, para lo cual al ser presentada al ISP se acompaña la información científica referida a:
- Estudios de desarrollo del producto farmacéutico, que incluyan estudios químicos, farmacéuticos y biológicos, según corresponda, de la fórmula propuesta y su justificación.

- Estudios pre-clínicos, tales como aquellos estudios que se realizan "in vitro" y/o en animales de experimentación, diseñados generalmente con la finalidad de obtener información necesaria para decidir si se justifican estudios más amplios en seres humanos, sin exponerlos a riesgos injustificados.

- Estudios farmacológicos selectivos en animales.

- Estudios toxicológicos en animales, que corresponderán a los ensayos de toxicidad aguda y crónica, teratogenicidad, embriotoxicidad, fertilidad, mutagénesis $\mathrm{y}$, en su caso, carcinogénesis y, en general, aquellos que sean necesarios para una correcta evaluación de la seguridad y tolerancia de un producto farmacéutico.

- Estudios clínicos fases I, II y III, que avalen la seguridad y eficacia del producto que se pretende registrar. Se podrán presentar estudios clínicos de productos que no correspondan a la fórmula que se pretenda registrar, siempre que se haya acreditado su equivalencia farmacéutica y terapéutica, mediante los respectivos estudios.

- Estudios farmacocinéticos, cuando corresponda.

- Estudios para demostrar biodisponibilidad o equivalencia terapéutica del producto farmacéutico que se pretenda registrar, en el caso de los productos cuyos principios activos estén sujetos a dicha exigencia. Los estudios de biodisponibilidad corresponden a estudios farmacocinéticos que, a través de un diseño experimental pre-establecido, permiten determinar la biodisponibilidad de un principio activo de una formulación que esté sujeta a un proceso de absorción. Por otra parte, los estudios de bioequivalencia corresponden a estudios clínicos que pretenden demostrar que la biodisponibilidad de un fármaco incorporado a una determinada formulación extravascular ("potencial genérico") es esencialmente la misma que la que presenta dicho fármaco incorporado en una forma farmacéutica de referencia (medicamento de marca), cuya patente ha expirado. La biodisponibilidad expresa la magnitud y la velocidad a la que un fármaco accede en forma inalterada al torrente circulatorio. Para su determinación se utilizan diferentes parámetros farmacocinéticos, siendo el área bajo la curva de concentraciones plasmáticastiempo (ABC) el utilizado para comparar la magnitud de la biodisponibilidad entre dos formulaciones, ya que este parámetro refleja el grado de exposición del organismo al fármaco y se considera una variable que subroga su respuesta terapéutica.

Son equivalentes farmacéuticos productos farmacéuticos que contienen idénticas cantidades de los mismos principios activos o sus mismas sales o ésteres, 
presentados en idéntica forma farmacéutica y vía de administración, pero que no necesariamente contienen los mismos excipientes y que cumplen con las mismas o comparables especificaciones de calidad.

Corresponden a equivalentes terapéuticos ${ }^{10}$, productos equivalentes farmacéuticos que cumplen con las mismas especificaciones de calidad y que al ser administrados según las condiciones especificadas en su rotulación sus efectos, con respecto a eficacia y seguridad, son esencialmente los mismos, todo ello determinado por estudios apropiados.

Los fármacos de administración intravascular se disuelven en la ampolla o frasco ampolla y al aplicarlos se distribuyen inmediatamente en el torrente sanguíneo con una biodisponibilidad de $100 \%$; no correspondiendo solicitar estudios de biodisponibilidad. Para certificar la calidad, seguridad y eficacia de una solución inyectable endovenosa los estudios recomendados por la Farmacopea de E.U.A. (USP) son los de pureza, valoración e identidad.

- Informe químico, farmacéutico y biológico, que incluya pruebas analíticas, químicas, fisicoquímicas, biológicas o microbiológicas y que permitan concluir si el producto que se pretende registrar se encuentra conforme con la composición declarada, presenta la adecuada calidad, los métodos de control propuestos se ajustan al estado de los conocimientos científicos, la formulación y forma farmacéutica están adecuados a los fines propuestos y el envase es idóneo para la correcta conservación.

- Informe toxicológico y farmacológico, que incluya pruebas o ensayos pre-clínicos que permitan reportar cuál es la toxicidad del producto y cuáles son sus propiedades farmacológicas comprobadas.

- Informe clínico elaborado por experto externo. El informe deberá evaluar los estudios aportados por el solicitante, en cuanto éstos puedan avalar que el producto demuestra una adecuada tolerancia, que la posología recomendada es correcta, así como las eventuales contraindicaciones y efectos secundarios.

\subsection{Artículo 32, de la calidad:}

- El laboratorio debe declarar proveedor y fabricante de él o los principios activos, adjuntando el boletín de análisis con todos los parámetros que lo caractericen.

- Condiciones de almacenamiento del principio activo como materia prima.

- Los antecedentes de estabilidad deben incluir como mínimo: fórmula estudiada, identificación del fabricante y responsable del estudio de estabilidad, condiciones de temperatura, humedad, material del envase y series estudiadas, además incluir el diseño programado y señalar los procedimientos analíticos utilizados y las especificaciones de producto terminado. Todo lo anterior, de acuerdo a la Guía de Estabilidad de Productos Farmacéuticos, aprobada como norma técnica mediante Decreto Supremo del Ministerio, a propuesta del ISP.

- Cuando el producto requiere dilución previa a su administración, se deberán adjuntar los estudios de estabilidad para la formulación, el solvente (si se incluye en la presentación) y el producto reconstituido. En el caso de que el producto requiera dilución y no se incluya en la presentación el solvente, se indicarán los solventes recomendados y se requerirá además la presentación de los estudios de estabilidad de la formulación y del producto reconstituido.

\subsection{Artículo 218, de la farmacovigilancia:}

Los titulares de registros o autorizaciones sanitarios deberán implementar y mantener un sistema de farmacovigilancia, propio o externalizado, siendo su Asesor Técnico, responsable de: crear, adoptar y mantener un sistema documentado para recopilar y tratar en un archivo único la información sobre todas las presuntas reacciones adversas.

Considerando la gran cantidad de documentación solicitada al momento de registro de un medicamento en nuestro país, se identifican los siguientes aspectos a ser considerados en los procesos de adquisición de antimicrobianos (ver cuadro).

\section{Glosario de términos}

\section{Decreto Supremo 3/2010. Artículo 5}

- Buenas Prácticas de Laboratorio (BPL): Conjunto de reglas, procedimientos operativos y prácticas que garantizan que los datos generados por un sistema de control de calidad son reproducibles y representativos, asegurando la validez y confiabilidad de los resultados; estas normas técnicas serán aprobadas por Decreto Supremo del Ministerio, a propuesta del ISP.

- Práctica de Buena Manufactura (PBM): Normas técnicas mínimas establecidas para todos los procedimientos destinados a garantizar la calidad uniforme y satisfactoria de los productos farmacéuticos, dentro de los límites aceptados y vigentes para cada uno de ellos; estas normas técnicas serán aprobadas por Decreto Supremo del Ministerio, a propuesta del ISP.

- Estudio de Equivalencia Terapéutica: Estudio comparativo (clínico, de biodisponibilidad, farmacodinámico o "in vitro") entre un producto farmacéutico de referencia o comparador y otro estudio.

- Estudio de Estabilidad: Serie de pruebas, ensayos y análisis relacionados con las características físicas, químicas o microbiológicas de un principio activo o un producto farmacéutico, para obtener información 


\begin{tabular}{|c|c|}
\hline Especificaciones técnicas a tener en cuenta & Fundamento \\
\hline Resolución del Instituto de Salud Pública-ISP de Registro Sanitario vigente & $\begin{array}{l}\text { Permiten demostrar que el producto cuenta con evaluación técnico-científica por parte } \\
\text { del organismo regulador del país; la que busca asegurar la calidad, seguridad y eficacia } \\
\text { de los medicamentos }\end{array}$ \\
\hline $\begin{array}{l}\text { Certificado de Práctica de Buena Manufactura (Good Manufacturing Practice-GMP) o } \\
\text { equivalente para la planta fabricante del principio activo }\end{array}$ & $\begin{array}{l}\text { Refleja el cumplimiento de las GMP indispensables para garantizar la calidad uniforme } \\
\text { y satisfactoria del principio activo manufacturado; dentro de los límites aceptados y } \\
\text { vigentes para cada uno de ellos. } \\
\text { Decreto Supremo 3/2010. Artículo 153, Artículo } 32^{\circ}\end{array}$ \\
\hline Certificado analítico del principio activo que identifique el fabricante y su origen & $\begin{array}{l}\text { Permite respaldar que el principio activo empleado en el producto farmacéutico cumple } \\
\text { con los parámetros de calidad establecidos. El fabricante y su origen } \\
\text { Decreto Supremo } 3 / 2010 \text {. Artículo } 36^{\circ}\end{array}$ \\
\hline $\begin{array}{l}\text { Certificado analítico del último lote del medicamento ofertado que identifique provee- } \\
\text { dor y procedencia principio activo }\end{array}$ & $\begin{array}{l}\text { Determina la trazabilidad del producto } \\
\text { Decreto Supremo } 3 / 2010 \text {. Artículo } 32^{\circ}\end{array}$ \\
\hline Folleto de información al profesional aprobado por el ISP & Indica las autorizaciones del medicamento en Chile \\
\hline $\begin{array}{l}\text { Para productos intravenosos presentar estudio de equivalencia farmacéutica y para } \\
\text { medicamentos de administración extravascular presentar estudios de bioequivalencia } \\
\text { entre el producto alternativo y el original realizado por un centro independiente que } \\
\text { cuente con la autorización del ISP }\end{array}$ & $\begin{array}{l}\text { Para principios activos sujetos a dicha exigencia } \\
\text { Decreto Supremo } 3 / 2010 \text {. Artículo } 36^{\circ}\end{array}$ \\
\hline $\begin{array}{l}\text { Certificado Food and Drug Administration-FDA y/o European Medicines Agency-EMA } \\
\text { u otra agencia regulatoria de referencia para el medicamento ofertado }\end{array}$ & $\begin{array}{l}\text { Demuestra la autorización para fines de comercialización del producto en cita, por } \\
\text { parte de las agencias regulatorias de referencia internacionales }\end{array}$ \\
\hline Presentar estudios clínicos (in vivo, in vitro, farmacocinética, farmacodinamia) & $\begin{array}{l}\text { Presentar estudios clínicos según corresponda (in vivo, in vitro, farmacocinética, far- } \\
\text { macodinamia) } \\
\text { Decreto Supremo } 3 / 2010 \text {. Artículo } 36^{\circ}\end{array}$ \\
\hline $\begin{array}{l}\text { Certificado de Práctica de Buena Manufactura o equivalente para la planta fabricante } \\
\text { del producto terminado }\end{array}$ & $\begin{array}{l}\text { Refleja el cumplimiento de las GMP indispensables para garantizar la calidad uniforme } \\
\text { y satisfactoria del producto terminado (todas las etapas-granel, envasado primario y } \\
\text { envasado secundario); dentro de los límites aceptados y vigentes para cada uno de ellos } \\
\text { Decreto Supremo } 3 / 2010 \text {. Artículo } 148 \text {, Artículo } 32^{\circ}\end{array}$ \\
\hline $\begin{array}{l}\text { Programa de farmacovigilancia para la totalidad de los medicamentos adjudicados } \\
\text { durante todo el período que dure el suministro }\end{array}$ & $\begin{array}{l}\text { Certificado que respalda la existencia de un sistema activo de farmacovigilancia } \\
\text { Decreto Supremo 3/2010. Artículo } 218\end{array}$ \\
\hline Reporte de fallas de calidad del medicamento en los últimos dos años & $\begin{array}{l}\text { Documento que certifica la inexistencia de fallas de calidad en los lugares en que se } \\
\text { comercializa } \\
\text { Decreto Supremo 3/2010 }\end{array}$ \\
\hline $\begin{array}{l}\text { Estabilidad de la solución reconstituida y diluida por cada solvente compatible a } T^{\circ} \\
\left(2-8^{\circ} \mathrm{C}\right),\left(25-32^{\circ} \mathrm{C} \text {, especificar) por un laboratorio certificado }\right.\end{array}$ & $\begin{array}{l}\text { Avala seguridad y eficacia } \\
\text { Decreto Supremo } 3 / 2010 \text {. Artículo } 32^{\circ}\end{array}$ \\
\hline
\end{tabular}

sobre su estabilidad, con el fin de definir su período de eficacia en determinadas condiciones de envase y almacenamiento.

- Estudio de Estabilidad a Tiempo Real: Estudio de estabilidad realizado por el tiempo propuesto para el período de eficacia y en condiciones de almacenamiento, temperatura y humedad determinadas según la naturaleza del producto.

- Estudio de Equivalencia Farmacéutica: Estudio aplicable a productos que contienen cantidades idénticas del mismo principio activo, en la misma forma farmacéutica, pero que no necesariamente contienen los mismos ingredientes inactivos. Se realiza un estudio comparativo químico y farmacéutico entre un producto farmacéutico innovador y un genérico para evaluar pureza, potencia, calidad e identidad.

\section{Taller 4}

La transmisión en el control de la resistencia, políticas de traslados, tamizaje y precauciones de contacto

\section{Introducción}

Para controlar el problema de resistencia bacteriana se sugieren medidas que se dividen fundamentalmente en el uso adecuado de los antimicrobianos para controlar la presión selectiva de éstos y preservar opciones de tratamiento, y en medidas enfocadas a controlar la transmisión horizontal o persona a persona de los microorganismos multi-resistentes (MOMR) en un medio principalmente hospitalario. Estas últimas corresponden a intervenciones de diversos tipos tales como medidas administrativas, educación del personal de salud y 
pacientes, vigilancia de MOMR, medidas de control de infecciones y control del ambiente ${ }^{1}$. En este taller se abordaron sólo algunas de estas intervenciones con énfasis en la vigilancia de MOMR en pacientes trasladados intentando proponer un protocolo consensuado para las instituciones nacionales.

\section{Vigilancia de MOMR}

Existen varias formas de realizarla:

- Detección a partir de los cultivos clínicos de rutina. Los cultivos clínicos detectan sólo una minoría de pacientes con MOMR. En pacientes que ingresan a unidades de cuidados intensivos se ha estimado una proporción de hasta $70 \%$ de E. coli y Klebsiella spp. productoras de BLEE no detectadas. En pacientes con cultivos clínicos y de vigilancia positivos, los cultivos de vigilancia han sido positivos en promedio 2,7 días antes que los clínicos ${ }^{2}$. De acuerdo a estos hallazgos, esta alternativa de vigilancia se considera sub-óptima.

- Vigilancia de agentes causales de infecciones asociadas a atención de salud (IAAS). Corresponde a la identificación y registro de MOMR que causan IAAS durante la realización de la vigilancia epidemiológica habitual. Es la forma de vigilancia que utiliza la mayoría de los centros hospitalarios de nuestro país de acuerdo a la normativa del Ministerio de Salud (MINSAL) de Chile y permite focalizar intervenciones y recursos en estos casos. Sin embargo, esta alternativa no identifica todos los pacientes con MOMR que pueden servir de reservorio y favorecer la transmisión.

- Detección de colonización por MOMR. Corresponde a la identificación de colonización por MOMR en un paciente, antes del desarrollo de una infección clínica. Es la intervención de mayor sensibilidad para detectar los casos y que tiene la mayor posibilidad de anticipar la implementación de medidas de control como precaución de contacto para disminuir el riesgo de transmisión de MOMR en las instituciones de salud. Sin embargo, existen múltiples barreras para considerar su implementación, siendo una de las principales el alto costo que implica. Otras limitaciones incluyen la falta de criterios claros para su implementación y la dificultad para demostrar su impacto en la disminución de tasas de IAAS en cada institución. Además en casos de alta colonización pudiesen haber dificultades en la implementación de medidas de control.

Los traslados de pacientes han demostrado ser una fuente importante en la transmisión de MOMR intra e interhospitales; por ello, en este taller se discutieron y consensuaron algunos aspectos de la vigilancia de colonización de pacientes trasladados que se discuten a continuación.
¿Es recomendable realizar vigilancia de colonización por MOMR en pacientes trasladados?

En la actualidad, es cada vez más frecuente recibir pacientes trasladados de otras instituciones por las redes de derivación de camas críticas, los servicios prestados por instituciones privadas a hospitales públicos y la complejidad cada vez mayor de los pacientes que atendemos. Esto genera un riesgo de trasmisión de MOMR entre las instituciones como ha quedado demostrado en varias situaciones recientes, incluso entre distintos países ${ }^{3-5}$. De acuerdo a lo señalado, la vigilancia activa de pacientes trasladados se considera en general (una medida recomendable, pero no aplicable necesariamente a todos los hospitales). Se debe tener presente la realidad local en cuanto a recursos y complejidad, y a la endemia de MOMR que puede ser muy distinta entre los distintos hospitales.

\section{¿Qué criterios se deben utilizar para definir estos pa- cientes?}

Los criterios deben depender de la realidad de la institución recomendando considerar:

- Pacientes complejos: con estadía en unidades de paciente crítico (UPC), neonatología, cirugía, sometidos a cirugía o procedimientos, portadores de dispositivos invasores al momento del traslado.

- Pacientes que han tenido hospitalización en el extranjero.

- Pacientes provenientes de centros de reposo y hospitales de crónicos.

- Además, puede plantearse realizar vigilancia de los traslados internos, especialmente desde unidades de alta complejidad a unidades de baja complejidad.

- Es clave la comunicación expedita y transparente entre las instituciones explicitando los hallazgos microbiológicos en los comentarios de alta o traslado.

\section{¿Qué microorganismos vigilar?}

Considerando que es complejo y de muy alto costo vigilar todos los MOMR, se propone vigilar sólo algunos de ellos. Además es importante recordar que se ha establecido que los pacientes comparten factores de riesgo para estar colonizados o infectados por $\mathrm{MOMR}^{6}$, por lo que algunos pueden considerarse marcadores de colonización. Se propone vigilar:

- Enterococcus resistente a vancomicina (ERV), el que se considera marcador de colonización por bacterias multi-resistentes en general, ya que los factores de riesgo son, en su mayoría, comunes con SARM y enterobacterias productoras de $\mathrm{BLEE}^{6}$. A modo de ejemplo, en un estudio en la Universidad de Maryland, E.U.A., se detectó que $47 \%$ de los pacientes colonizados por enterobacterias productoras de BLEE estaban cocolonizados por ERV, y que si no se detectaba portación de ERV, el valor predictor negativo de portación de enterobacterias productoras de BLEE era de $99 \%{ }^{2}$. 
- Staphylococcus aureus resistente a meticilina.

- Sólo se propone ampliar la vigilancia a bacilos gramnegativos multi-resistentes $\mathrm{u}$ otros agentes dependiendo de la realidad de cada centro.

\section{¿Qué sitios de muestreo son los recomendados?}

De acuerdo a las guías internacionales vigentes ${ }^{7-9}$, los sitios recomendados son:

- SARM: fosas nasales (narinas).

- $E R V$ : hisopado rectal o materia fecal; esta segunda muestra, de preferencia en pacientes intensamente neutropénicos.

- Bacilos gramnegativos: no hay un sitio de tamizaje o tipo de muestra estandarizado; puede ser cultivo de aspirado endotraqueal en pacientes intubados o hisopado rectal. La zona inguinal ha sido recomendada en la guía más reciente publicada en $2014^{9}$.

\section{¿Qué método utilizar para la vigilancia?}

- Los métodos de biología molecular (reacción de polimerasa en cadena-RPC) demuestran claramente mayor sensibilidad, pero el costo de implementación y proceso es claramente mayor. El menor tiempo de respuesta compensa, en parte, los costos al disminuir el tiempo de uso de los implementos para precaución de contacto en espera de los resultados ${ }^{10,11}$.

- Los cultivos tradicionales pueden ser adecuados para comenzar un programa de vigilancia en hospitales menos complejos, que no dispongan de métodos moleculares o que tengan presupuestos muy reducidos.

- La utilización de medios cromogénicos comerciales, disponibles para SARM, ERV y enterobacterias productoras de BLEE disminuyen el tiempo de respuesta.

\section{Propuesta de protocolo de vigilancia de pacientes trasladados}

- Consiste en la instalación de precaución de contacto y toma de muestras para SARM y ERV a pacientes trasladados desde otros centros asistenciales que cumplan al menos uno de los siguientes criterios:

- Presencia de procedimientos invasores con excepción de catéter venoso periférico, independiente del tiempo de hospitalización.

- Antecedentes de haber sido sometido a cirugía durante la hospitalización actual (previa al traslado), independiente del tiempo de hospitalización.

- Hospitalización actual (previa al traslado) mayor a $72 \mathrm{~h}$.

- Hospitalización actual (previa al traslado) en Unidades de Paciente Crítico o Intermedio, independiente del tiempo de hospitalización.

- Antecedentes de hospitalización por más de $72 \mathrm{~h}$ en los tres meses previos al ingreso.
- Para SARM se realiza hisopado nasal (narinas) y para ERV hisopado rectal.

- Ambos exámenes se procesan por RPC en laboratorio de urgencia para que estén disponibles en forma continua.

- El resultado estará disponible aproximadamente en tres horas.

- Si el resultado de los RPC es negativo y no hay otros cultivos o estudios pendientes que lo ameriten, se suspende la precaución de contacto.

\section{Taller 5}

\section{Recomendaciones para los establecimientos} de salud frente a informe de paciente con enterobacteria productora de carbapenemasa

El objetivo de este taller fue sugerir aquellas medidas que deben ser implementadas en cualquier institución cuando se aísla una enterobacteria resistente a carbapenémico, mediada por la presencia de un gen que produce carbapenemasa, sea ésta de tipo KPC (Klebsiella pneumoniae carbapenemase) $\mathrm{u}$ otra.

Las enterobacterias pueden ser resistentes a carbapenémicos por dos mecanismos principales: uno de bajo nivel, mediado por la hiper-expresión de AmpC y/o BLEE asociado a pérdida de algunas porinas y el segundo, mediado por enzimas que inactivan carbapenémicos (carbapenemasa). En Chile, el mecanismo más frecuente descrito como causa de resistencia de enterobacterias a carbapenémicos, es el mediado por perdida de porinas más la presencia de una enzima inactivadora de cefalosporinas; sin embargo, cuando la resistencia a carbapenémicos es mediada por carbapenemasas, existe mayor trascendencia clínica y epidemiológica ya que se anula la posibilidad de tratamiento con todos los carbapenémicos y tiene un potencial de diseminación mucho mayor.

Se define una enterobacteria productora de carbapenemasa a aquella bacteria de la familia Enterobacteriaceae que presenta susceptibilidad disminuida a carbapenémicos, mediada por actividad de carbapenemasas. La detección de enterobacterias productoras de carbapenemasas tiene un impacto clínico considerable ya que usualmente está asociada a la presencia de muchos otros determinantes de resistencia, siendo estas bacterias habitualmente extremadamente resistentes (XDR) o incluso pan-resistentes.

Los estudios de susceptibilidad por los diferentes métodos detectan la susceptibilidad disminuida a los carbapenémicos pero, a través de ellos, no se puede inferir la presencia de carbapenemasa. La detección de las carbapenemasas basada sólo en los valores de CIM o en los milímetros de halos de inhibición a carbapenémicos definidos como puntos de corte, tanto en el CLSI como 
EUCAST no tienen sensibilidad óptima, existiendo cepas productoras de carbapenemasas que caen en la categoría de intermedio e incluso sensibles. Por lo anterior, la búsqueda de carbapenemasas debiese ser realizada en cualquier enterobacteria con cualquier grado de disminución en la susceptibilidad a carbapenémicos ${ }^{22}$.

Actualmente no existe consenso qué CIM o halo debiese aplicarse para investigar la presencia de carbapenemasas. En la literatura científica se ha recomendado realizarlo con valores $\geq 0,5 \mu \mathrm{g} / \mathrm{mL}$ para ertapenen; $\geq 1 \mu \mathrm{g} /$ $\mathrm{mL}$ para imipenem y meropenem ${ }^{23}$ o con halos $<25 \mathrm{~mm}$ en ertapenem y meropenem y $<23$ en imipenem ${ }^{24}$.

Aquella cepa sospechosa de producir carbapenemasa debiera ser sometida a un test de hidrólisis de carbapenémicos. Si el test de hidrólisis es positivo, es muy posible que esa cepa tenga presencia de carbapenemasa, la cual habría que determinar con estudios moleculares. Existen diferentes métodos para evaluar hidrólisis de carbapenémico. Se sugiere como método simple de realizar para la detección de carbapenemasas en cualquier laboratorio, el ensayo de hidrolisis de carbapenémicos "Carba NP" (por el nombre de sus creadores). Este test supera en sensibilidad y especificidad al test de Hodge modificado y al de ácido fenil borónico y además, es de rápida lectura $(2 \mathrm{~h})$, por lo que podría ser implementado en cualquier laboratorio clínico ${ }^{24}$. En Chile este test es realizado en diversos centros. Un resultado positivo de este test necesariamente debe llevar a un estudio molecular en búsqueda de una carbapenemasa específica. La confirmación de la presencia del gen de carbapenemasa debe ser realizada por biología molecular por lo que una cepa sospechosa de acuerdo a criterios anteriores, debe ser siempre derivada al Centro de Referencia Nacional. Existe actualmente una normativa que indica los criterios para derivación de cepas sospechosas que incluye resultados de CIM y de mm de diámetro del halo, sin considerar aún, los test de hidrólisis ${ }^{25}$.

En Chile no existe endemia de Enterobacteriaceae resistentes a carbapenémicos mediada por carbapenemasas, y los casos descritos a la fecha (no más de 30), han sido aislados. Sin embargo, países cercanos como Brasil, Colombia y Argentina tienen una alta endemia y, frente a pacientes que hayan estado hospitalizados en algunos de esos países, pareciera razonable descartar su presencia como colonizante, así como cualquier paciente proveniente de otros países más lejanos que poseen endemia por este tipo de enzimas.

Dado la situación epidemiológica actual en Chile, consideramos que las medidas a continuación sugeridas, deben ser implementadas cuando:

- El paciente tiene antecedentes epidemiológicos de haber estado en un país con endemia.

- Hay un aislamiento de una enterobacteria resistente a uno o más carbapenémicos.
- Y se ha realizado un test de hidrólisis que ha dado positivo.

En este caso, el laboratorio debe notificar al equipo de IAAS quienes deben tener previamente protocolizadas las medidas a tomar. Pueden dividirse éstas en ${ }^{26-32}$ :

- Administrativas: Enfocadas a mantener el flujo de información desde y hacia las autoridades del hospital de manera de garantizar el financiamiento y transversalidad de las medidas.

- Enfocadas en el paciente caso: Aquellas que garanticen la correcta aplicación de las precauciones de contacto sin descuidar el la calidad de la atención del paciente.

- Enfocadas en los contactos: Tendientes a evitar la diseminación o aparición no detectada de casos secundarios.

- Personal de salud: Asegurarán la información y capacitación del personal de salud para lograr el adecuado cumplimiento de las medidas instauradas.

- Ambientales: Tendientes a disminuir la probabilidad de persistencia del agente en el ambiente facilitando su erradicación.

- Epidemiológicas: Enfocadas en la detección de portación y seguimiento en el tiempo.

Se ha definido como fundamentales, las siguientes medidas (ver cuadro)

\section{Conclusiones de los Talleres 2014}

La resistencia bacteriana ofrece una epidemiología cambiante por lo que se hace necesaria la revisión periódica de las conductas y procedimientos frente a este tema, tanto desde el punto de vista diagnóstico, terapéutico, epidemiológico como del control de su diseminación. Referente al diagnóstico y pruebas de susceptibilidad antimicrobiana, el CLSI evalúa y hace recomendaciones anualmente de acuerdo a criterios preestablecidos y de aplicación local en E.U.A. Dada la calidad del panel de expertos sus recomendaciones son seguidas en América Latina y en particular en Chile. A pesar de ello, no todas las recomendaciones, pueden ser aplicadas en nuestro país y se requiere un análisis crítico de las mismas.

- En el primer taller de discusión se analizaron las dificultades en la implementación local de las últimas recomendaciones del CLSI y las opciones que se podrían aplicar en nuestro país según la epidemiología y conocimientos locales.

- En cuanto a los datos epidemiológicos de resistencia bacteriana, hasta la fecha, disponemos de información de porcentajes de susceptibilidad para diversos agentes, provista por los laboratorios de microbiología de 


\begin{tabular}{|c|c|c|c|c|c|c|c|c|c|c|}
\hline & 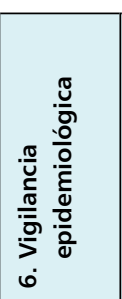 & 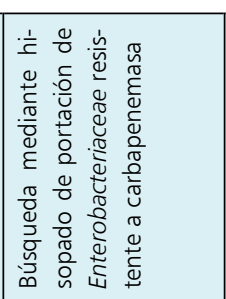 & 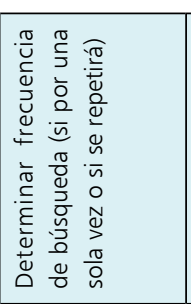 & 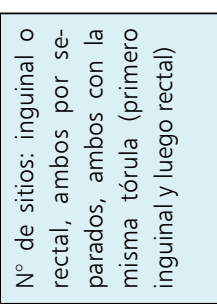 & 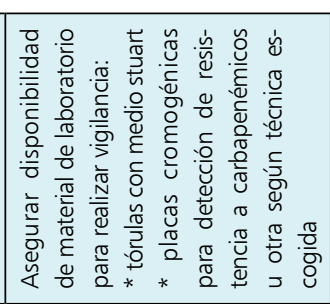 & 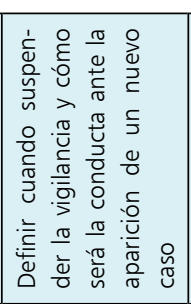 & & & & \\
\hline & \multicolumn{10}{|c|}{ 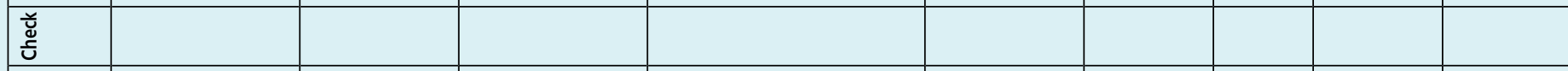 } \\
\hline$\frac{8}{8}$ & 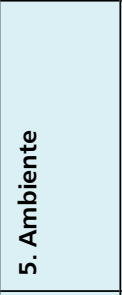 & 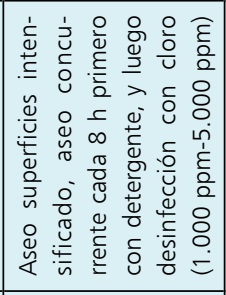 & 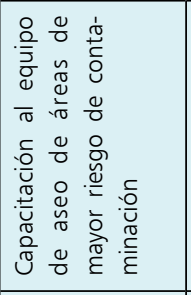 & 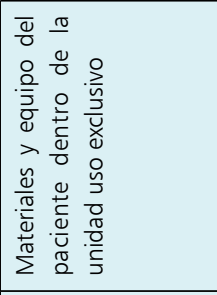 & 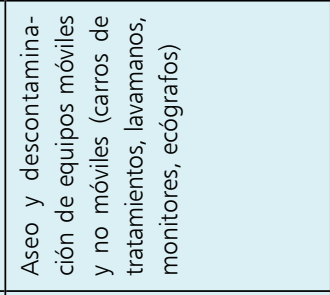 & 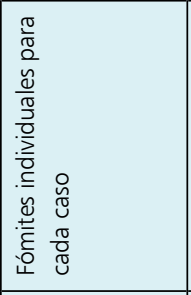 & 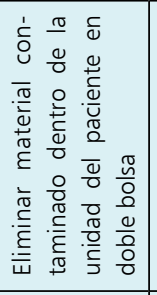 & 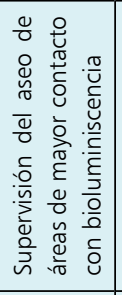 & 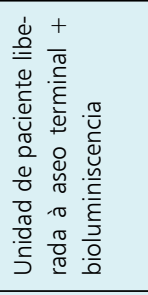 & 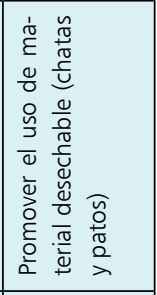 \\
\hline \multicolumn{11}{|c|}{ 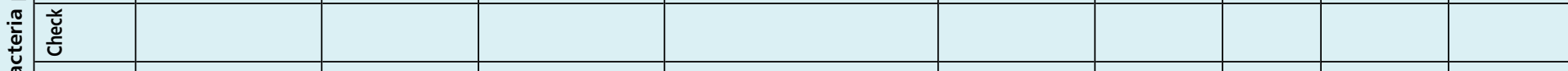 } \\
\hline 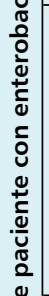 & 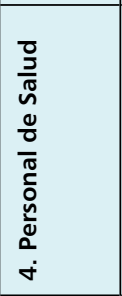 & 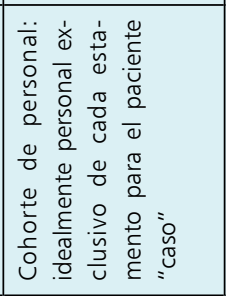 & 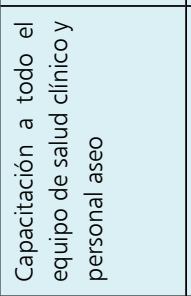 & 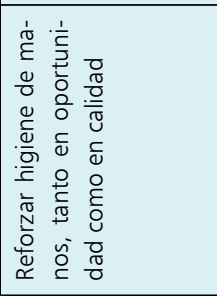 & 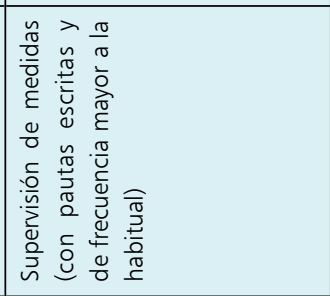 & 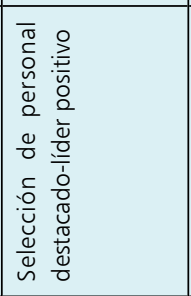 & & & & \\
\hline & 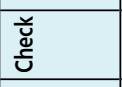 & & & & & & & & & \\
\hline \multirow{2}{*}{ 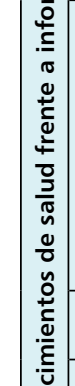 } & 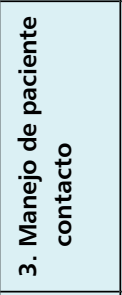 & 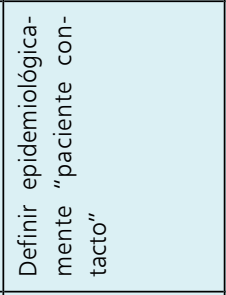 & 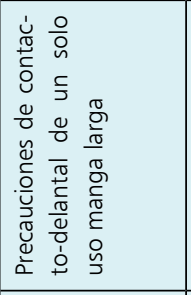 & 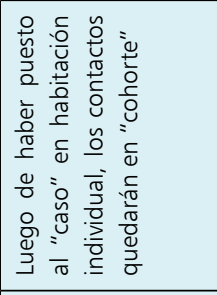 & 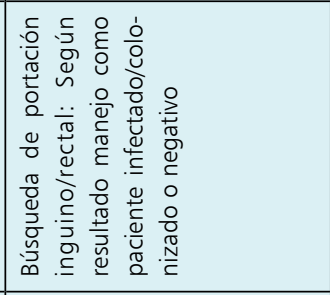 & 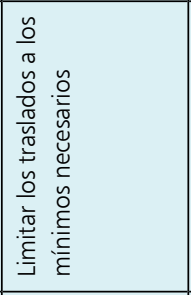 & 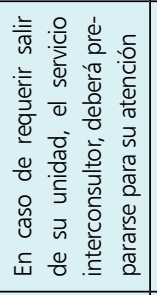 & 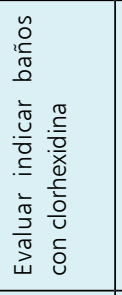 & & \\
\hline & 蒙 & & & & & & & & & \\
\hline & 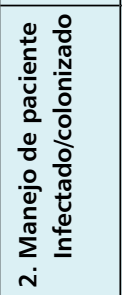 & 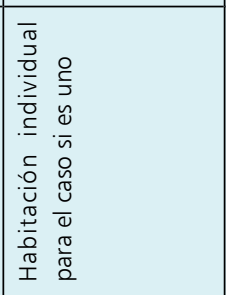 & 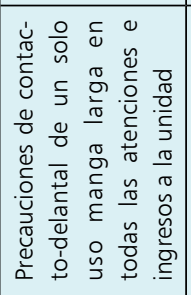 & 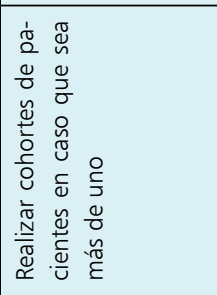 & 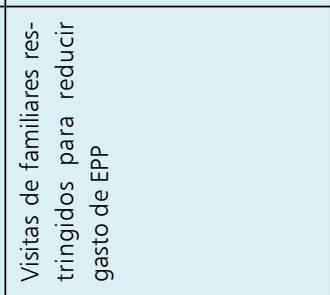 & 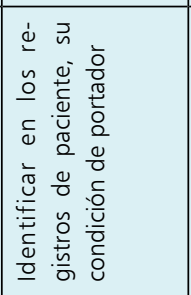 & 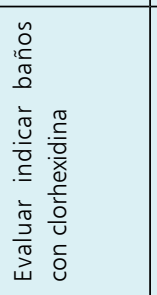 & 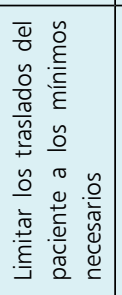 & 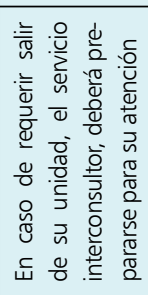 & 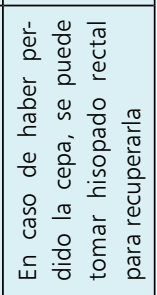 \\
\hline & 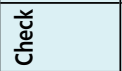 & & & & & & & & & \\
\hline 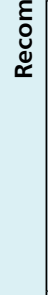 & 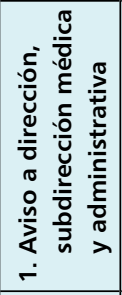 & 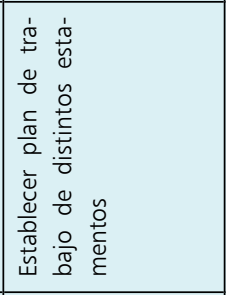 & 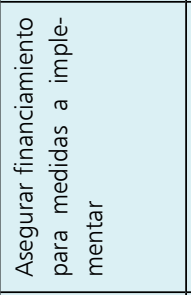 & 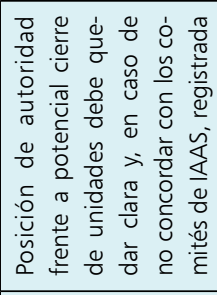 & 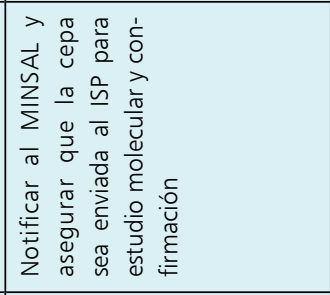 & & & & & \\
\hline & $\begin{array}{l}\stackrel{\check{\ddot{u}}}{\mathrm{~g}} \\
\end{array}$ & & & & & & & & & \\
\hline
\end{tabular}


manera estandarizada y en un número considerable de instituciones de nuestro país. Sin embargo, los porcentajes de susceptibilidad no necesariamente reflejan el problema epidemiológico de un hospital. En ese sentido, el grupo de participantes del Taller 2, sugiere complementar esta información con la medición de la "densidad de incidencia", la cual refleja de mejor manera la real endemia de bacterias con resistencia antimicrobiana de importancia epidemiológica y, eventualmente, en algunas instituciones además podría entregar datos del impacto clínico de estos microorganismos.

- Entre los microorganismos de alta importancia epidemiológica figuran, de manera especial, las enterobacterias con resistencia a carbapenémicos mediada por carbapenemasas, que constituyen una amenaza de salud pública para nuestros hospitales y para la cual aún estamos en condiciones de detener su diseminación. Aquellos hospitales que han tenido pacientes portadores o infectados con enterobacterias productoras de carbapenemasas han adaptado e implementado rápidamente las medidas de control recomendadas por la literatura científica, con éxito en la erradicación, ya que no se ha observado endemia o brotes posteriores a su detección y manejo. En el Taller 5, se analizaron las recomendaciones en base a la experiencia de estos hospitales y la evidencia que la respalda y se generó una lista de chequeo de fácil aplicación en caso de detección de nuevos casos.

- Si consideramos el tratamiento de bacterias multiresistentes, una necesidad frecuente para los infectólogos es conocer los requisitos de calidad que se deben solicitar a los antimicrobianos genéricos que nos permitan recomendar a nuestros hospitales medicamentos seguros y eficaces que pudieran ser comparables a los innovadores (originales). En pacientes graves, asegurar que el fármaco elegido es igualmente efectivo que el innovador es crítico para el éxito clínico; por ende, se deben conocer todos aquellos puntos fundamentales para la exigencia al administrador de salud. En el Taller número 3 se mencionan varios de estos puntos considerando los requisitos administrativos, legales y farmacológicos necesarios para asegurar la mejor calidad y seguridad para los pacientes.

- En los países en vías de desarrollo, como el nuestro, la transmisión de bacterias resistentes entre pacientes de diferentes hospitales es particularmente importante por la gran movilidad de los mismos, especialmente en los hospitales de la red pública. Consideramos relevante estandarizar el manejo que debieran tener los pacientes que se trasladan entre diferentes establecimientos ya que pueden ser portadores de microbiota diferente a la del hospital de destino del paciente. El taller que abordó este tema, establece sugerencias para el tamizaje de patógenos y de precauciones de contacto frente a esta circunstancia.

Consideramos que los temas analizados en los talleres de la última reunión del GCR, si bien abordan puntos específicos y acotados sobre resistencia bacteriana, reflejan una necesidad que ha sido manifestada en distintas instancias por los participantes. Dado la importancia epidemiológica, creemos necesario compartir con la comunidad médica los acuerdos alcanzados. Destacamos que esto no representa una posición oficial nacional, ni un consenso de Sociedades Científicas ni de otras entidades. Las conclusiones de los participantes del Grupo Colaborativo de Resistencia Bacteriana sólo representan la experiencia local y la revisión de la literatura científica realizada, de modo que cada institución pueda evaluar de mejor manera las medidas importantes de implementar según su epidemiología y realidad local.

Dado que los cambios de resistencia bacteriana son dinámicos, progresivos e incluso vertiginosos, es importante considerar que muchas de las recomendaciones aquí compartidas, pueden requerir modificaciones en un futuro cercano. Así, estimamos que es muy importante reevaluar periódicamente estas sugerencias, así como también incluir otros temas epidemiológicamente relevantes en resistencia bacteriana que no han sido incluidos en este documento. Hasta la fecha, en el mundo, la resistencia bacteriana ha ido aumentando como un evento difícil de controlar. Sólo el trabajo conjunto, integrado y coordinado en cada institución nacional, puede contribuir a retardar o disminuir esta amenaza de salud pública.

\section{Resumen}

En la reunión anual del Grupo Colaborativo de Resistencia Bacteriana del año 2014 se revisaron en profundidad cinco tópicos cuyos antecedentes y conclusiones se detallan en este documento. Los temas fueron: I.-Novedades del CLSI 2014: se revisaron las dificultades e implicancias de su implementación a nivel local y se establecen recomendaciones. II.- Criterios para la determinación de incidencia de microoganismos multi-resistentes en unidades de pacientes críticos, donde se definieron los indicadores y la metodología de vigilancia para una mejor cuantificación del problema. III.-Se establecieron requisitos de calidad a considerar por los profesionales que participan en la selección de antimicrobianos en el hospital. IV.- Se discutieron las políticas de traslado, tamizaje y precauciones de contacto para el control de la transmisión de bacterias multiresistentes. V.- Se establecieron recomendaciones para los establecimientos de 
salud frente a la pesquisa de una enterobacteria productora de carbapenemasa en formato de lista de chequeo para la implementación rápida en hospitales sin endemia de estos agentes. Estas sugerencias nacen del trabajo conjunto de especialistas de muchos hospitales, no representan un consenso o normativa pero pueden ser de ayuda para el control de la resistencia en cada establecimiento de salud del país.

\section{Referencias bibliográficas}

\section{Taller 1}

1.- CLSI Performance Standards for Antimicrobial Susceptibility Testing; Twenty-fourth Informational Supplement, CLSI document M100-S24. Wayne, PA: Clinical and Laboratory Standards Institute 2014.

2.- Livermore D M, Andrews J M, Hawkey P M, Ho P L, Keness Y, Doi Y, et al. Are susceptibility tests enough, or should laboratories still seek ESBLs and carbapenemases directly? J Antimicrob Chemother 2012; 67 (7): 1569-77.

3.- Esparza G, Ariza B, Bedoya A M, Bustos I, Castañeda-Ramírez C, De la Cadena E, et al. Estrategias para la implementación y reporte de los puntos de corte CLSI vigentes y pruebas fenotípicas confirmatorias para BLEE y carbapenemasas en bacilos Gram negativos en laboratorios clínicos de Colombia. Infect 2013; 17 (2): 80-9.

Taller 2

4.- Cohen A L, Calfee D, Fridkin S K, Huang S S, Jernigan J A, Lautenbach E, et al. For the Society for Healthcare Epidemiology of America and the Healthcare Infection Control Practices Advisory Committee. Recommendations for Metrics for MultidrugResistant Organisms in Healthcare Settings: SHEA/HICPAC Position Paper. Infect Control Hosp Epidemiol 2008; 29 (10).

5.- Furuya E Y, Larson E, Landers T, Jia H, Ross B, Behta M. Challenges of Applying the SHEA/HICPAC Metrics for MultidrugResistant Organisms to a Real-World Setting. Infec Control and Hosp Epidemiol 32 (4): 323-32.

6.- Magiorakos A P, Srinivasan A, Carey R B, Carmeli Y, Falagas M E, Giske C G, et al. Multidrug-resistant, extensively drug-resistant and pandrug-resistant bacteria: an international expert proposal for interim standard definitions for acquired resistance. Clin Microbiol Infect 2012; 18: 268-81.

7.- O'Brien T F, Stelling J M. WHONET: an information system for monitoring antimicrobial resistance. Emerg Infect Dis 1995; 1 (2): 66.

8.- O'Brien T F, Stelling J M. Integrated multilevel surveillance of the world's infecting microbes and their resistance to antimicrobial agents. Clin Microbiol Rev 2011; 24 (2): 281-95.
Taller 3

9.- D.S. No 3/2010: Reglamento del Sistema Nacional de Control de Productos Farmacéuticos de Uso Humano, publicado en el Diario Oficial el 25 de junio de 2011 y vigente a contar del 26 de diciembre de 2011. "Norma General Técnica sobre sistema de farmacovigilancia de productos farmacéuticos de uso humano". En http://web.minsal.cl/portal/ url/item/c4a31ad6db50e085e040010165017a39. pdf

10.- Norma que define los criterios destinados a establecer equivalencia terapéutica en productos farmacéuticos en Chile. Resolución Exenta $\mathrm{N}^{\circ}$ 727/05. http://www.ispch.cl/sites/default/files/ decreto_exento_27.pdf

\section{Taller 4}

11.- Siegel J D, Rhinehart E, Jackson M, Chiarello L. Healthcare Infection Control Practices Advisory Committee. Management of MDROs in healthcare setting. CDC Guideline 2006. Am J Infect Control 2007; 35 (Suppl 2): S 65-164.

12.- Harris A D, Nemoy L, Johnson J A, Martin-Carnahan A, Smith D L, Standiford $\mathrm{H}$, et al. Co-carriage rates of vancomycin-resistant enterococcus and extended-spectrum beta-lactamase-producing bacteria among a cohort of intensive care unit patients: implications for an active surveillance program. Infect Control Hosp Epidemiol 2004; 25: $105-8$

13.- Cifuentes M, García P, San Martín P, Silva F, Zúñiga $\mathrm{J}$, Reyes $\mathrm{S}$, et al. Primer caso de detección de blaKPC en Chile: desde Italia a un hospital público de Santiago. Rev Chilena Infectol 2012; 29 (2): 224-8.

14.- Yong D, Toleman M A, Giske C G, Cho H S, Sundman K, Lee K, et al. Characterization of a new metallo-beta-lactamase gene, blaNDM-1, and a novel erythromycin esterase gene carried on a unique genetic structure in Klebsiella pneumoniae sequence type 14 from India. Antimicrob Agents Chemother 2009; 53: 5046-54.

15.- Halaby T, Reuland A E, Al Naiemi N, Potron A, Savelkoul P H, VandenbrouckeGrauls C M, et al. A Case of New Delhi Metallo- Lactamase 1 (NDM-1)-Producing Klebsiella pneumoniae with putative secondary transmission from the Balkan Region in the Netherlands. Antimicrob Agents Chemother 2012; 56 (5): 2790-1.
16.- Safdar N, Maki D. The commonality of risk factors for nosocomial colonization and infection with antimicrobial-resistant Staphylococcus aureus, Enterococcus, gram-negative bacilli, Clostridium difficile, and Candida. Ann Intern Med 2002; 136 (11): 834-44.

17.- Muto C A, Jernigan J A, Ostrowsky B E, Richet H M, Jarvis W R, Boyce J M, et al. SHEA Guideline for preventing nosocomial transmission of multidrug-resistant strains of Staphylococcus aureus and Enterococcus. Infect Control Hosp Epidemiol 2003; 24: 362-86.

18.- Siegel J D, Rhinehart E, Jackson M, Chiarello L, and the Healthcare Infection Control Practices Advisory Committee, 2007 Guideline for Isolation Precautions: Preventing Transmission of Infectious Agents in Healthcare Settings http://www.cdc.gov/ncidod/dhqp/pdf/ isolation2007.pdf

19.- Taconelli E, Cataldo M A, Dancer S J, De Angelis G, Falcone M, Frank U, et al. ESCMID Guideline for the management of the infection control measures to reduce transmission of MDR-GNB in hospitalized patients. Clin Microbiol Infect 2014; 20 (Suppl. I): 1-55.

20. Diekema D J, Pfaller M A. Rapid detection of antibiotic-resistant organism carriage for infection prevention. Rapid detection of antibiotic-resistant organism carriage for infection prevention. Clin Infect Dis 2013; 56 (11): 1614-20.

21.- Shenoy E S, Kim J, Rosenberg E S, Cotter J A, Lee H, Walensky R P, et al. Discontinuation of contact precautions for methicillin-resistant Staphylococcus aureus: A randomized controlled trial comparing passive and active screening with culture and polymerase chain reaction. Clin Infect Dis 2013; 57 (2): 176-84.

Taller 5

22.- Nordmann P, Poirel L, Dortet L. Rapid detection of carbapenemase-producing Enterobacteriaceae. Emerg Infect Dis 2012; 18 (9): 1503-7.

23.- Nordmann P, Poirel L. Strategies for identification of carbapenemase-producing Enterobacteriaceae. J Antimicrob Chemother 2013; 68: 487-9.

24.- European Committee on Antimicrobial Susceptibility Testing. EUCAST guidelines for 
detection of resistance mechanisms and specific resistances of clinical and/or epidemiological importance EUCAST, December 2012.

En: http://www.eucast.org/fileadmin/src/ media/PDFs/EUCAST_files/Consultation/ EUCAST_guidelines_detection_of_resistance mechanisms_121222.pdf

25.- Circular $N^{\circ} 01 / 2013$ Ministerio de Salud. Instrucciones para la vigilancia nacional de resistencia a los antimicrobianos de importancia epidemiológica en bacterias que pueden producir infecciones asociadas a la atención en salud (IAAS). Vigilancia de Resistencia en Enterobacterias. Enero de 2013.

26.- Institute National de Santé Publique du Quebéc. Comité sur les infections nosocomiales du Québec. 2010. Prévention et controle de la transmission des entérobacteries productricies de carbapénemases dans les mileaux de soins aigus du Québec. 2010. En www.inspq.qc.ca/
pdf/publications/1168_PreventionTransmission EnterobactCarbapenemases.pdf

27.- Australian Commission on Safety and Quality in Health Care. Recommendations for the control of Multi-drug resistant Gram-negatives: carbapenem resistant Enterobacteriaceae (Oct 2013). Sydney. ACSQHC, 2013.

28.- Public Health England, UK. Acute trust toolkit for Early Detection, Management and control of carbapenemase producing Enterobacteriaceae. London. 2013. En https://www.gov.uk/government/ uploads/system/uploads/attachment_data/ file/329227/Acute_trust_toolkit_for_the_ early_detection.pdf

29.- Centers for Diseases Control. National Center for Emerging and Zoonotic Infectious Diseases. DHQP. Guidance for Control of Carbapenemresistant Enterobacteriaceae (CRE)-2012 CRE Toolkit. En www.cdc.gov/hai/pdfs/cre/CRE- guidance-508.pdf

30.- Centers for Diseases Control. Healthcareassociated Infections (HAIs). Guidelines for control of infections with carbapenem-resistant or carbapenemase-producing Enterobacteriaceae in acute care facilities. MMWR Morbid Mortal Wkly Rep 2009; 58 (10): 256-60.

31.- European Centre for Disease Prevention and Control. Carbapenemase-producing bacteria in Europe: interim results from the European Survey on carbapenemase-producing Enterobacteriaceae (EuSCAPE) project. Stockholm: ECDC; 2013.

32.- European Centre for Disease Prevention and Control. Risk assessment on the spread of carbapenemase-producing Enterobacteriaceae (CPE) through patient transfer between healthcare facilities, with special emphasis on cross-border transfer. Stockholm: ECDC; 2011. 